\title{
Pearson's syndrome without marrow involvement
}

\author{
A A M Morris, P J Lamont, P T Clayton
}

\begin{abstract}
A child with a mitochondrial DNA deletion who presented with pancreatic exocrine insufficiency is reported. Though she developed many other features of Pearson's syndrome, there was no bone marrow involvement. Syndromes associated with mitochondrial DNA defects are highly variable and absence of one feature should not inhibit investigation.

(Arch Dis Child 1997;77:56-57)
\end{abstract}

Keywords: Pearson's syndrome; mtDNA deletion; pancreatic exocrine insufficiency

Pearson's syndrome was originally defined as the presence of refractory sideroblastic anaemia with vacuolisation of marrow precursors and exocrine pancreatic dysfunction. ${ }^{1}$ Further experience has revealed other features including lactic acidaemia, renal tubular dysfunction, hepatic failure, diabetes mellitus, enteropathy and rashes $^{2}$; cases surviving beyond the first few years may develop features of KearnsSayre syndrome. ${ }^{2}$ Mitochondrial DNA (mtDNA) deletions have been identified in all cases, sometimes associated with other mtDNA rearrangements. ${ }^{2}$ The clinical pattern varies considerably, presumably reflecting the degree of heteroplasmy (that is the proportions of normal and rearranged mtDNA) in different tissues. The label Pearson's syndrome has been applied to a number of patients without pancreatic involvement. ${ }^{2}$ Here we report a child with many features of Pearson syndrome, who lacked marrow involvement.

\section{Case report}

Our patient, the first child of healthy parents, was well until aged 6 months, when she started to have recurrent episodes of diarrhoea. Between 16 and 20 months of age, her stools were persistently bulky, loose, pale, and offensive and her weight fell from 8.2 to $7.0 \mathrm{~kg}$.

Investigations at 20 months showed raised blood lactate concentrations $(2.3,3.1,3.4,4.8$, and $5.0 \mathrm{mmol} / 1$, normal $<2.0$ ), with raised lactate:pyruvate ratios $(>35$, normal $<25)$. The cerebrospinal fluid lactate concentration was $4.1 \mathrm{mmol} / 1$ (normal <2.0). The plasma alanine and aspartate transaminase levels were raised at $71 \mathrm{IU} / 1$ (normal <45) and $164 \mathrm{IU} / 1$ (normal $<55)$ respectively, with normal clotting studies, alkaline phosphatase and bilirubin levels. There was a low plasma magnesium concentration $(0.45 \mathrm{mmol} / 1$, normal $0.74-1.00)$, generalised aminoaciduria and a raised urinary $N$-acetyl glucosaminidase/creatinine ratio (103 units/mmol, normal <27) but urinary calcium/ creatinine and albumin/creatinine ratios and the maximum tubular phosphate reabsorption were normal.

Aspiration of duodenal juice showed low levels of lipase and trypsin after cholecystokinin (lipase activity $228 \mu \mathrm{mol} / \mathrm{min} / \mathrm{ml}$, normal 360-1900; trypsin $23 \mu \mathrm{mol} / \mathrm{min} / \mathrm{ml}$, normal 50-220; assays as previously reported ${ }^{3}$ ). The bicarbonate concentration after secretin was also low ( $<5 \mathrm{mmol} / \mathrm{l}$, normal 46-85). Duodenal and jejunal biopsy specimens showed normal villus architecture but colonic specimens showed oedema of the lamina propria with markedly increased eosinophils. Muscle histology and histochemistry were normal. There was extensive hepatic steatosis, particularly around the central veins, with mild perivenular fibrosis. Cytochrome oxidase activity in hepatocytes was slightly lower than in controls.

Blood counts were consistently normal and a bone marrow trephine specimen taken at 20 months was normocellular with plentiful megakaryocytes and normal maturation of erythroid and myeloid precursors.

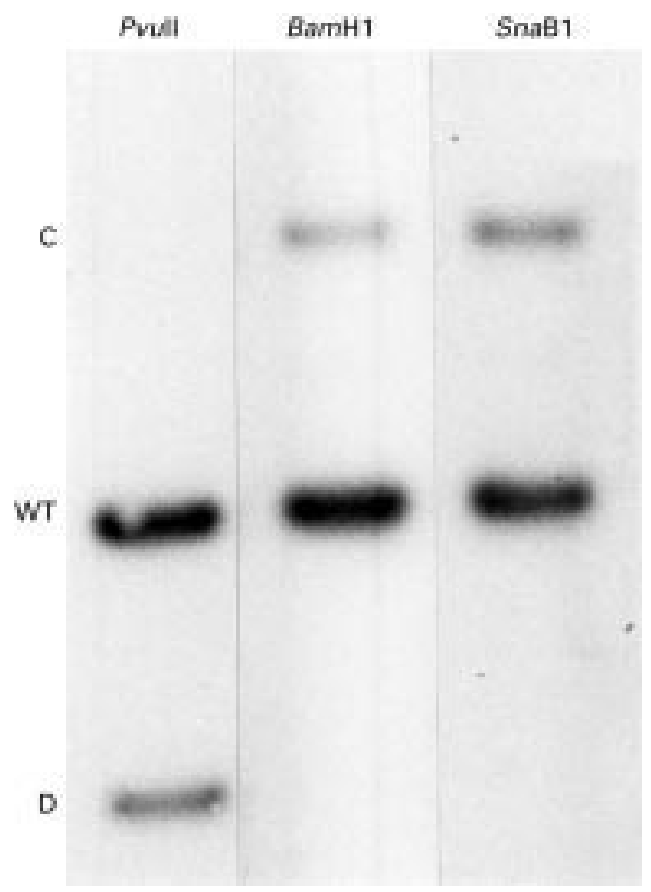

Figure 1 Southern blot analysis of $m t D N A$ from the patient's muscle after digestion with PvuII, Bam $\mathrm{H} 1$ and SnaB1. In the PvuII digest, the bands correspond to linearised wild-type (WT) and linearised deleted mtDNA (D). In the Bam $\mathrm{H} 1$ and SnaB1 digests, the bands correspond to linearised wild-type mtDNA and intact circular deleted mtDNA (C), implying that the sites for these restriction enzymes have been lost. As circular molecules migrate slower than linearised ones, the bands corresponding to deleted mtDNA in these digests lie above those for linearised wild-type mtDNA. 
The patient was treated with magnesium, bicarbonate, and pancreatic enzyme supplements. In six months she gained $4.4 \mathrm{~kg}$, bringing her weight almost to the 25 th centile (the same centile as her length). At the age of 26 months, she developed a photosensitive rash and hypoparathyroidism (ionised calcium 0.75 $\mathrm{mmol} / \mathrm{l}$, normal 1.17-1.35; phosphate 2.56 $\mathrm{mmol} / 1$, normal 1.29-1.78; parathyroid hormone $0.7 \mathrm{pmol} / 1$, normal $1.0-6.5)$. Currently, at 30 months, neurodevelopmental examination and cranial magnetic resonance imaging remain normal.

\section{Molecular studies}

DNA was extracted from muscle and leucocytes and digested with the restriction endonucleases PvuII, BamH1, and SnaB1. The digested DNA fragments were separated by agarose gel $(0.6 \%)$ electrophoresis, transferred to a nylon membrane by Southern blotting and hybridised with a total mtDNA probe as previously described. ${ }^{4}$ The mtDNA fragments were visualised by autoradiography.

A heteroplasmic $5 \mathrm{~kb}$ deletion of mtDNA was demonstrated in muscle (fig 1) and in leucocytes (data not shown), associated with loss of the BamH1 and SnaB1 restriction sites but not the PvuII site. The proportion of deleted mtDNA appeared similar in muscle and leucocytes.

\section{Discussion}

This case illustrates the fact that mtDNA rearrangements can cause a wide spectrum of diseases in infancy and early childhood, including presentations which do not fulfil the criteria originally used to define Pearson's syndrome. mtDNA rearrangements should be considered in all cases of unexplained pancreatic exocrine insufficiency, even in the absence of anaemia. Pointers to the diagnosis included the raised lactate concentrations, hepatic and renal tubular abnormalities. Hepatic and renal involvement, however, may also accompany pancreatic disease in Shwachman syndrome. ${ }^{5}$ Moreover, in our patient, blood lactate concentrations were very variable, sometimes only being marginally above the upper limit of normal. One should, therefore, have a low threshold for seeking mtDNA rearrangements, which may be detectable in blood even if there are no haematological abnormalities.

We thank Dr R S Brown for referring the patient and for continuing help with her care.

1 Pearson HA, Lobel JS, Kocoshis SA, et al. A new syndrome of refractory sideroblastic anaemia with vacuolization of marrow precursors and exocrine pancreatic dysfunction. 7 marrow precursors and

2 Rotig A, Bourgeron T. Chretien D, Rustin P, Munnich A. Spectrum of mitochondrial DNA deletions in the Pearson marrow-pancreas syndrome. Hum Mol Genet 1995;4:132730 .

3 McCollum JPK, Muller DPR, Harries JT. Test meal for assessing intraluminal phase of absorption in children. Arch Dis Child 1977;52:887-9.

4 Holt IJ, Harding AK, Petty RKH, Morgan-Hughes JA. A new mitochondrial disease associated with mitochondrial DNA heteroplasmy. Am F Hum Genet 1990;46:428-33.

5 Aggett PJ, Cavanagh NP, Matthew DJ, Pincott JR, Sutcliffe J, Harries JT. Shwachman syndrome. Arch Dis Child 1980; 55:331-47. 